\title{
WORST CASE ANALYSIS OF A SIMPLE MIMO MASS-SPRING-DAMPER SYSTEM
}

\author{
Mark Halton* Martin J. Hayes ${ }^{* \dagger}$ Petar Iordanov ** \\ * Department of Electronic and Computer Engineering, \\ University of Limerick, Limerick, IRELAND \\ ** Department of Electronic Engineering, NUI Maynooth, \\ Maynooth, IRELAND \\ † E-mail:martin.j.hayes@ul.ie
}

\begin{abstract}
This paper considers the application of the structured singular value, $\mu$, and the skewed structured singular value, abbreviated "skew $\mu$ ", to the analysis of a (deceptively) simple MIMO mass-spring-damper system. This example highlights some pertinent outstanding $\mu$ analysis questions, namely the effect of strictly real, possibly repeated parameters and the limitation of classical grid-based methods. It is shown that by transforming the original $\mu$ problem and using skew $\mu$ to calculate the worst case that more reliable and certifiably safe answers to the analysis question are determined. A comparison with more conventional $\mu$ analysis methods is presented. Copyright (C)2005 IFAC
\end{abstract}

Keywords: robust stability, uncertainty, structured singular value

\section{INTRODUCTION}

The study of multivariable systems has now reached the stage where reliable answers to the "limit of performance" question are now required. In particular for system models that allow for parameter variation or uncertainty, the engineer wants to know the vector of real physical parameters that will cause the worst case to occur. This paper considers the use of the structured singular value, $\mu$, and the skewed structured singular value, abbreviated "skew $\mu$ ", for a system where the associated parameter uncertainty is constrained to be real. It is well known that the $\mu$ analysis of this type of system provokes significant computational difficulties for commercial software packages. This becomes quickly apparent to many first-time users of the $\mu$-Tools Toolbox (Balas et al., 1998)) when performing an analysis on a simple second order mass-spring-damper system. The $\mu$-Tools lower bound algorithm of (Young and
Doyle, 1997) fails to converge when the uncertainty set is constrained to be real. This is a major cause of concern, mainly because no useful worst case problem perturbation will be returned by the analysis. Of course, since the classic mass-springdamper system is SISO, other methods to determine the worst case are feasible and may be more appropriate. However, a straightforward extension to a MIMO double mass-spring-damper (MSD) system can render many conventional analysis techniques essentially useless. It is well known that techniques based on gain margin or phase margin cannot be used to assess stability for the MIMO case (Doyle and Stein, 1981). In this paper, the so-called " $\mu$-paradigm" for the computation of the worst case (or maximum gain) of an uncertain double MSD system is considered. In particular, the merits and drawbacks for the analysis question where the uncertainty is quite naturally constrained to be strictly real is discussed. In this fashion quite subtle limitations in existing 
$\mu$ analysis software are highlighted quickly in an accessible fashion. It is shown that a certifiably safe method for the computation of the worst case can be obtained by transforming the problem to include frequency as a real uncertain parameter in the problem formulation. The analysis then becomes a single skew $\mu$ computation on a constant matrix which is inherently attractive. An optimization-based skew $\mu$ algorithm developed in (Halton, 2004) is used to determine good lower bound information. It is shown that by using this approach a problem perturbation is returned which is valid for a given user defined frequency interval. The skew $\mu$ upper bound algorithm of (Holland et al., 2003) is used to determine if any solution found using the lower bound algorithm is local or global, and to ensure no higher value of $\mu$ exists over an entire frequency range.

This paper is outlined as follows, section 2 introduces the necessary nomenclature and details formal definitions for both $\mu$ and skew $\mu$. Section 3 introduces the double MSD model and associated notation while in section 4 three popular (lower bound) approaches used to determine worst case solutions for the real $\mu$ problem are reviewed. Section 5 details the necessary transformation and optimisation-based techniques used to reliably determine the worst case. Results and conclusions are detailed in section 6 and section 7 respectively.

\section{ROBUSTNESS ANALYSIS TECHNIQUES}

The $\mu$ approach for systems analysis is based on the observation that problems involving interconnections of LTI systems with uncertain parameters and unmodelled dynamics can be reduced to considering the constant matrix feedback interconnection in figure 1 . The uncertainty block $\Delta$ is structured where three non-negative integers $m_{r}, m_{c}$ and $m_{C}$ specify the number of uncertainty blocks of each type. The block structure $\mathcal{K}\left(m_{r}, m_{c}, m_{C}\right)$ is an $m$-tuple of positive integers:

$$
\begin{aligned}
& \mathcal{K}=\left(k_{1}, \ldots, k_{m_{r}}, k_{m_{r}+1}, \ldots, k_{m_{r}+m_{c}}, \ldots\right. \\
& \left.\ldots k_{m_{r}+m_{c}+1}, \ldots, k_{m}\right)
\end{aligned}
$$

with $m=m_{r}+m_{c}+m_{C}$. This $m$-tuple specifies the dimensions of the perturbation blocks, which determines the set of allowable perturbations, namely define:

$$
\begin{aligned}
X_{\mathcal{K}}=\{ & \Delta=\text { block } \operatorname{diag}\left(\delta_{1}^{r} I_{k_{1}}, \ldots, \delta_{m_{r}}^{r} I_{k_{m_{r}}}, \ldots\right. \\
& \left.\left.\delta_{1}^{c} I_{k_{m_{r}+1}}, \ldots, \delta_{m_{c}}^{c} I_{k_{m_{r}+m_{c}}}, \Delta_{1}^{C}, \ldots, \Delta_{m_{C}}^{C}\right)\right\}
\end{aligned}
$$

with:

$$
\delta_{i}^{r} \in \mathbf{R}, \delta_{i}^{c} \in \mathbf{C}, \Delta_{i}^{C} \in \mathbf{C}^{k_{m_{r}+m_{c}+i} \times k_{m_{r}+m_{c}+i}}
$$

Note that $X_{\mathcal{K}} \subset \mathbf{C}^{n \times n}$ (where $n=\sum_{i=1}^{m} k_{i}$ ) and that this block structure allows for repeated

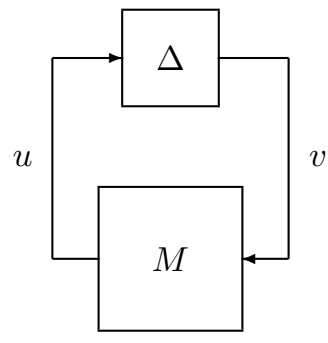

Fig. 1. Canonical $\mu$ analysis framework

real scalars $\left(\delta_{i}^{r} I\right)$, repeated complex scalars $\left(\delta_{i}^{c} I\right)$, and full complex blocks $\left(\Delta_{i}^{C}\right)$. Noting this block structure, the following definition, taken from (Doyle, 1982) is introduced.

Definition 2.1. The structured singular value, $\mu_{\mathcal{K}}(M)$, of a matrix $M \in \mathbf{C}^{n \times n}$ with respect to a block structure $\mathcal{K}\left(m_{r}, m_{c}, m_{C}\right)$ is defined as:

$$
\mu_{\mathcal{K}}(M)=\frac{1}{\min _{\Delta \in X_{\mathcal{K}}}\left\{\bar{\sigma}(\Delta): \operatorname{det}\left(I_{n}-\Delta M\right)=0\right\}}
$$

with $\mu_{\mathcal{K}}(M)=0$ if no $\Delta \in X_{\mathcal{K}}$ solves $\operatorname{det}\left(I_{n}-\right.$ $\Delta M)=0$. Linear Fractional Transformations (LFTs) are used to reorganize a perturbed problem with uncertainty into the feedback interconnection in figure 1. In particular, if $M \in \mathbf{C}^{n \times n}$ is partitioned as:

$$
M=\left[\begin{array}{ll}
M_{11} & M_{12} \\
M_{21} & M_{22}
\end{array}\right]
$$

with $M_{11} \in \mathbf{C}^{n_{1} \times n_{1}}, M_{22} \in \mathbf{C}^{n_{2} \times n_{2}}$ and $n=n_{1}+$ $n_{2}$, then an upper LFT will be described as:

$$
\Delta * M=M_{22}+M_{21} \Delta\left(I_{n_{1}}-M_{11} \Delta\right)^{-1} M_{12}
$$

If two block structures are defined as $\mathcal{X}_{\mathcal{K}_{1}} \subset$ $\mathbf{C}^{n_{1} \times n_{1}}, \mathcal{X}_{\mathcal{K}_{2}} \subset \mathbf{C}^{n_{2} \times n_{2}}$, then the augmented block structure $\mathcal{X}_{\hat{\mathcal{K}}} \in \mathbf{C}^{n \times n}$ is defined as:

$\mathcal{X}_{\hat{\mathcal{K}}}=\left\{\Delta=\operatorname{block} \operatorname{diag}\left(\Delta_{f}, \Delta_{v}\right):\right.$

$$
\left.\Delta_{f} \in \mathbf{B} \mathcal{X}_{\mathcal{K}_{1}}, \Delta_{v} \in \mathcal{X}_{\mathcal{K}_{2}}\right\}
$$

where $\mathbf{B} \mathcal{X}_{\mathcal{K}_{1}}=\left\{\Delta_{f} \in \mathcal{X}_{\mathcal{K}_{1}}: \bar{\sigma}\left(\Delta_{f}\right) \leq 1\right\}$. The skewed structured singular value is the smallest structured singular value of a subset of perturbations that destabilizes the system $M$ with the remainder of the perturbations contained within a fixed range. Formally stating this

Definition 2.2. The skewed structured singular value, $\mu_{\hat{\mathcal{K}}}^{s}(M)$, of a matrix $M \in \mathbf{C}^{n \times n}$ with respect to a block structure $\hat{\mathcal{K}}\left(m_{r_{f}}, m_{c_{f}}, m_{C_{f}}, m_{r_{v}}, m_{c_{v}}, m_{C_{v}}\right)$ is defined as:

$$
\mu_{\hat{\mathcal{K}}}^{s}(M)=\frac{1}{\min _{\Delta \in \mathcal{X}_{\hat{\mathcal{K}}}}\left\{\bar{\sigma}\left(\Delta_{v}\right): \operatorname{det}\left(I_{n}-\Delta M\right)=0\right\}}
$$

with $\mu_{\hat{\mathcal{K}}}^{s}(M)=0$ if no $\Delta \in \mathcal{X}_{\hat{\mathcal{K}}}$ solves $\operatorname{det}\left(I_{n}-\right.$ $\Delta M)=0$. 


\section{LOWER BOUND ALGORITHMS}

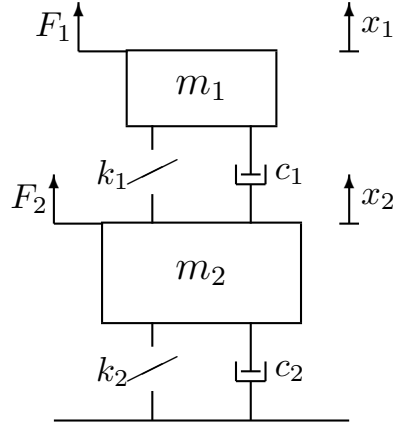

Fig. 2. MIMO mass-spring-damper model

\section{MOTIVATING EXAMPLE}

Consider an apparently simple double MSD in figure 2. The forces on the masses are the inputs and their respective displacements are the outputs. The state and input matrices are derived as:

$A=\left[\begin{array}{cccc}0 & 0 & 1 & 0 \\ 0 & 0 & 0 & 1 \\ -\frac{k_{1}}{m_{1}} & \frac{k_{1}}{m_{1}} & -\frac{c_{1}}{m_{1}} & \frac{c_{1}}{m_{1}} \\ \frac{k_{1}}{m_{2}} & -\frac{k_{1}+k_{2}}{m_{2}} & \frac{c_{1}}{m_{2}} & -\frac{c_{1}+c_{2}}{m_{2}}\end{array}\right], B=\left[\begin{array}{cc}\frac{1}{m_{1}} & 0 \\ 0 & \frac{1}{m_{2}} \\ 0 & 0 \\ 0 & 0\end{array}\right]$

This is a popular example in control theory and is an excellent illustrative example of a relatively simple MIMO system. The question arises for the vector of physical parameters $m_{i}, c_{i}$ and $k_{i}$ which are fixed but uncertain is "how is it possible to reliably assess the robust stability of this system?". From a $\mu$ analysis perspective, it should be noted that the type of uncertainty that is necessary to represent the variation in the physical parameters will only be real valued. It is also necessary to emphasize that the uncertainty associated with the physical parameters will be repeated due to the reoccurrence of the same parameter multiple times in the state equations. In order to assess the robustness of the system using $\mu$, an LFT must be generated. The associated perturbation block contains both real repeated and nonrepeated uncertain parameters and is given by:

$$
\Delta=\operatorname{diag}\left(\delta_{m_{1}}^{r}, \delta_{m_{2}}^{r}, \delta_{c_{1}}^{r} I_{2}, \delta_{c_{2}}^{r}, \delta_{k_{1}}^{r} I_{2}, \delta_{k_{2}}^{r}\right)
$$

In this canonical form, it is possible to carry out a classical $\mu$ analysis using a frequency grid. The problem with any approach of this kind is that it has been shown in (Barmish et al., 1990) that real $\mu$ is discontinuous as a function of frequency. This means that if a point discontinuity exits, it may be missed due to the nature of the grid-based search. For an illustrative example, see (Sideris, 1992). It should also be noted that analytical methods to determine potential frequency points where real $\mu$ is discontinuous become quickly infeasible for large perturbation sets.
To obtain a better lower bound on real $\mu$ and/or a worst case problem perturbation, three different frequency-based methods are now discussed. These include an approach where the worst case is assumed to occur at some combination of extreme values, an approach outlined in (Dailey, 1990), a regularization method detailed in (Packard and Pandey, 1993) and an optimization-based method presented in (Iordanov, 2003).

\subsection{Worst Case at Extremities?}

Undergraduate linear programming texts suggest that, in general, the worst case problem perturbation can be obtained at the extremities of the allowable range of variation for each uncertain variable. These extremities can be considered as vertices of a "box" in the parameter space. An obvious drawback is that the number of combinations that need to be checked for each addition of an uncertain parameter grows at $2^{n}$ where $n$ is the number of uncertain parameters. Consequently, the search for a worst case solution is exponential time and soon becomes infeasible for large perturbation blocks.

\subsection{Dailey's Method}

In the search to determine a worst case problem perturbation associated with real $\mu$, Dailey's algorithm is often used (Dailey, 1990). Based on Dailey's conjecture, the search for finding a solution to:

$$
\operatorname{det}\left(I_{n}-\Delta M(s)\right)=0
$$

reduces to solving only two uncertain parameters $\delta_{i}^{r}$ and $\delta_{j}^{r}$ at any given time. Using a combination of matrix algebra and a number of determinant identities, determining a solution for equation 4 reduces to finding the real roots of a set of two quadratic equations in $\delta_{i}^{r}$ and $\delta_{j}^{r}$. If the solutions are complex, then no real solutions exist. Although proposed as a possible method to potentially determine the exact value of $\mu$, this technique provides a lower bound since counterexamples exist (Holohan and Safonov, 1993).

\subsection{Regularized $\mu$ Lower Bound}

A lower bound for real $\mu$ that can be computed in polynomial time, denoted as a regularization method, is proposed (Packard and Pandey, 1993). This approach, which is suggested in the $\mu$-Tools manual as a solution to the real $\mu$ problem, "regularizes" the original $\mu$ problem by essentially adding a small amount of complex uncertainty 
to each real parameter. By doing this, a small amount of phase uncertainty is added to the gain uncertainty. It is subsequently shown that the new mixed $\mu$ problem is continuous unlike its real $\mu$ counterpart. Given $M \in \mathbf{C}^{n \times n}$ and the block structures $\mathcal{X}_{\mathcal{K}_{1}} \subset \mathbf{R}^{n \times n}$ and $\mathcal{X}_{\mathcal{K}_{2}} \subset \mathbf{C}^{n \times n}$, the algorithm is based on the following theorem.

Theorem 4.1. (Regularized $\mu$ )

Let $0<\epsilon \in \mathbf{R}$. Suppose that $M(s)$ has all of its poles in the open left-half-plane (i.e. nominal stability) and let $\beta>0$. Given $X_{\mathcal{K}}$ compatible with $M(s)$, define a new uncertainty structure $\mathcal{X}_{\hat{\mathcal{K}}}$ as:

$\mathcal{X}_{\hat{\mathcal{K}}}=\left\{\right.$ block $\left.\operatorname{diag}\left(\Delta_{1}, \Delta_{2}\right): \Delta_{1} \in \mathcal{X}_{\mathcal{K}_{1}}, \Delta_{2} \in \mathcal{X}_{\mathcal{K}_{2}}\right\}$

Then for all $\Delta \in \mathcal{M}\left(\mathcal{X}_{\hat{\mathcal{K}}}\right)$ with $\|\Delta\|_{\infty} \leqslant \beta$, the perturbed closed-loop system is (well-posed and) uniformly stable if and only if:

$$
\mu_{\hat{\mathcal{K}}}\left(\left[\begin{array}{cc}
\frac{1}{\beta} M & \frac{\sqrt{\epsilon}}{\beta} M \\
\frac{\sqrt{\epsilon}}{\beta} M & \frac{\epsilon}{\beta} M
\end{array}\right]\right)<1
$$

where $\mu_{\hat{\mathcal{K}}}(\cdot)$ is continuous for the frequency interval $\omega \in[0, \infty]$.

\subsection{Optimization-based $\mu$ Lower Bound}

An optimization-based lower bound solution for real $\mu$ that works well on relatively large practical applications and potentially returns more useful worst case destabilizing information is presented in (Iordanov, 2003). For consistency of notation, it is now stated in a slightly different form.

Theorem 4.2. (Optimized $\mu$ lower bound)

Let $0 \leq \emptyset_{d} \in \mathbf{R}$. For $M \in \mathcal{C}^{n \times n}$ and any compatible block structure $\mathcal{K}\left(m_{r}, 0,0\right)$, a lower bound on $\mu_{\mathcal{K}}(M)$ can be determined from:

$$
\mu_{\mathcal{K}}(M)=\frac{1}{\min _{\Delta \in X_{\mathcal{K}}}\left\{\|\Delta\|:\left|\operatorname{det}\left(I_{n}-\Delta M\right)\right| \leq \emptyset_{d}\right\}}
$$

where the solution is (the inverse of) a constrained minimization optimization problem.

\subsection{Merits/Drawbacks of Each Approach}

Dailey's conjecture is a very attractive approach but like the search over all extremities, the algorithm is exponential time since it is necessary to search each 2-dimensional face of a $n$-dimensional hypercube. This limits the algorithm's usefulness to low order perturbation blocks. A more significant drawback is the fact that only nonrepeated real uncertain variables can be tolerated in the perturbation block. Therefore, it cannot be used on, for example, the MIMO mass-springdamper system. An important benefit of the regularization-based solution is that the original, potentially discontinuous, strictly real problem is transformed into a continuous mixed one. A further benefit is that the $\mu$-Tools algorithms may be used to determine the bounds on $\mu$ in polynomial time. However, a drawback of this approach is the selection of $\epsilon$ and tests must be performed a priori to ensure that the regularized model still represents the original system satisfactorily. A further drawback is the increase in the original problem size. In fact, the regularized perturbation block is double the size of the original. It can be shown that worst case problem perturbations returned are dominated by the complex entries and extracted real solutions are, in general, not valid. Consequently, no worst case information is obtained. The optimization-based method has many advantages over the regularization-based approach. No transformations are required and it has been shown in (Iordanov, 2003) that improved lower bound solutions are obtained. A further advantage is that useful explicit worst case information is returned from the unwrapped problem perturbation. Two drawbacks exist, the first is a feature of the algorithm and the second is a feature of the approach. Since any optimizationbased approach is heavily reliable on it initial starting solution, a number of iterations may be necessary to determine the best lower bound solution for $\mu$ at each frequency point. If a minimum number of 10 restarts are necessary, this means that the computation time increases by a factor of 10 over its $\mu$-Tools counterpart. Regardless, the solution search remains polynomial time. The second drawback is that the uncertainty block remains real, therefore it remains a grid-based approach and is potentially unreliable.

\section{A SKEW $\mu$ APPROACH}

The following theorem extends results first presented in (Sideris, 1992).

Theorem 5.1. (state-space $\mu$ )

Suppose that $M(s)$ has all of its poles in the open left-half-plane and let $\beta>0$. Given a minimal state-space representation of $M(s)$ and given $X_{\mathcal{K}}$ compatible with $M(s)$, define a new uncertainty structure $\mathcal{X}_{\hat{\mathcal{K}}}$ as:

$\mathcal{X}_{\hat{\mathcal{K}}}=\left\{\operatorname{block} \operatorname{diag}\left(\delta_{\omega}^{r} I_{p}, \Delta\right): \delta_{\omega}^{r} \in \mathbf{R}, \Delta \in X_{\mathcal{K}}\right\}$

then for all $\Delta \in \mathcal{M}\left(X_{\mathcal{K}}\right)$ with $\|\Delta\|_{\infty} \leqslant \beta$, the perturbed closed loop system is uniformly stable if and only if:

$$
\mu_{\hat{\mathcal{K}}}(H)<1
$$

where:

$H=\left[\begin{array}{cc}j \alpha\left(A-j \omega_{0} I_{p}\right)^{-1} & \sqrt{\frac{\alpha}{\beta}}\left(A-j \omega_{0} I_{p}\right)^{-1} B \\ -j \sqrt{\frac{\alpha}{\beta}} C\left(A-j \omega_{0} I_{p}\right)^{-1} & -\frac{1}{\beta}\left(C\left(A-j \omega_{0} I_{p}\right)^{-1} B-D\right)\end{array}\right]$ 
with $\omega_{0}=(\bar{\omega}+\underline{\omega}) / 2$ and $\alpha=(\bar{\omega}-\underline{\omega}) / 2$.

A full description of the this approach is presented in (Halton, 2004) where it is shown that this transformation may be recast as a skew $\mu$ problem where frequency is the skewed parameter. It is now possible to obtain the worst case perturbation from this test and the value of $\delta_{\omega}$ containing the worst case frequency information. Furthermore, this information may be determined or unwrapped using the following expression for this approach:

$$
s=j\left(\omega_{0}+\alpha \delta_{\omega}\right)
$$

Using this transformation, an optimization-based approach may be used to determine a lower bound on skew $\mu$ where the uncertainty is constrained to be real.

Theorem 5.2. (Optimized skew $\mu$ lower bound) Let $0 \leq \emptyset_{d} \in \mathbf{R}$. For $M \in \mathcal{C}^{n \times n}$ and any compatible block structure $\hat{\mathcal{K}}\left(m_{r_{f}}, 0,0, m_{r_{\nu}}, 0,0\right)$, a lower bound on $\mu_{\hat{\mathcal{K}}}^{s}(M)$ can be determined from:

$$
\mu_{\hat{\mathcal{K}}}^{s}(M)=\frac{1}{\min _{\Delta \in X_{\hat{\mathcal{K}}}}\left\{\left\|\Delta_{\nu_{l}}\right\|:\left|\operatorname{det}\left(I_{n}-\Delta M\right)\right| \leq \emptyset_{d}\right\}}
$$

where the solution is (the inverse of) a constrained minimization problem.

In order to obtain quality solutions, it is necessary to relax the nonlinear equality constraint in equation 2.1 to an inequality with the introduction of $\emptyset_{d}$ to counteract the non-convex nature of the problem search. Note that " $\emptyset_{d}$ " is the digital implementation of zero and is generally of magnitude $10^{-8}$ or less. The uncertainty block structure is implemented where one optimization variable represents a real-valued uncertainty parameter. This lower bound algorithm has been developed in Matlab using the Optimization Toolbox (Branch and Grace, 1996) and may be used with the statespace formulation to counteract the discontinuity issue associated with real $\mu$, and provide valid problem perturbations. An upper bound on skew $\mu$ may be obtained using the generalized eigenvalue formulation of (Holland et al., 2003) and is quantified in the following theorem.

Theorem 5.3. (Mixed skew $\mu$ upper bound) For $M \in \mathcal{C}^{n \times n}$ and any compatible block structure $\hat{\mathcal{K}}$, a skew $\mu$ upper bound, $\nu_{u}$, can be calculated from:

$$
\left(\mathcal{A}+\frac{1}{\nu_{u}} \mathcal{B}\right)\left[\begin{array}{l}
x_{1} \\
x_{2} \\
x_{3}
\end{array}\right]=0
$$

where:

$$
\mathcal{A}=\left[\begin{array}{cc|c}
M_{11} M_{11}^{*}-I_{f} & M_{11} M_{21}^{*} & 0 \\
M_{21} M_{11}^{*} & M_{21} M_{21}^{*} & I \\
\hline 0 & I & 0
\end{array}\right]
$$

and:

$$
\mathcal{B}=\left[\begin{array}{cc|c}
M_{12} M_{12}^{*} & M_{12} M_{22}^{*} & 0 \\
M_{22} M_{12}^{*} & M_{22} M_{22}^{*} & 0 \\
\hline 0 & 0 & I
\end{array}\right]
$$

In its current format, the formulation in equation 8 does not detail or consider standard $D$ and $G$ scaling matrices associated with calculation of the standard $\mu$-Tools upper bound, reflecting the structure of the associated perturbation block. It is detailed in (Halton, 2004) how this scaling is considered and implemented. Both upper and lower bound algorithms are available in beta form and will be implemented as part of the next release of the freely downloadable "MuExplorer" software (Iordanov, 2003). The performance of each approach is now illustrated on the double MSD example.

\section{RESULTS}

Using the Matlab $\mu$-Tools Toolbox, it is possible to assess the robust stability characteristics of the system for the arbitrary values and levels of uncertainty given in figure 2 . The resulting bound(s) on $\mu$ are shown in figure 3 . The $\mu$-Tools lower bound algorithm failed to return any solution hence no lower bound is plotted. The $\mu$ upper bound plot illustrates the danger of a frequency grid approach, displaying a very sharp peak for the frequency response. Using a regularization-based approach has the attractive feature of transforming the problem into a mixed uncertainty problem. This ensures the problem is continuous and the $\mu$-Tools lower bound algorithm may be used to obtain improved solutions. The lower bound plot shown is for $1 \%$ scaled complex uncertainty. Over the apparent critical range of $[0.1,10] \mathrm{rad} / \mathrm{s}$, the bound is extremely poor. Moreover, increasing the percentage uncertainty changes the transformed problem significantly from the original. Indeed, even for frequency points where the bound is tight, no worst case information is obtained from the extracted real uncertain variables. Irrespective, the result from the frequency sweep indicates that the system is robustly stable for the introduced levels of uncertainty.

Transforming the problem using equation 5.1, upper and lower skew $\mu$ bounds may be obtained (corresponding to the upper and lower bounds on the peak value of $\mu$ ) over predefined frequency intervals. The decades corresponding to the critical range of $[0.1,10] \mathrm{rad} / \mathrm{s}$ are intuitively subdivided into 10 sub-intervals. Both upper and lower bounds are included in figure 3 . The peak value of $\mu$ alarmingly exceeds unity for the interval $[0.8,0.9] \mathrm{rad} / \mathrm{s}$, verifying that the system is not robustly stable. It is evident that the solution 
Table 1. Unwrapped uncertain values.

\begin{tabular}{llllll}
\hline$\delta_{m_{1}}^{r}$ & $\delta_{m_{2}}^{r}$ & $\delta_{c_{1}}^{r}$ & $\delta_{c_{2}}^{r}$ & $\delta_{k_{1}}^{r}$ & $\delta_{k_{2}}^{r}$ \\
\hline 0.8066 & 0.1353 & -0.9342 & 0.9274 & -1.000 & 0.5871 \\
\hline \hline$m_{1}$ & $m_{2}$ & $c_{1}$ & $c_{2}$ & $k_{1}$ & $k_{2}$ \\
\hline 4.2099 & 6.4059 & 0.7197 & 1.4436 & 1.200 & 4.9394 \\
\hline
\end{tabular}

Table 2. Robust stability results.

\begin{tabular}{llll}
\hline Description & Points/Intervals & Max $\mu$ & Freq \\
\hline$\mu$-Tools UB & 300 points & 0.9210 & 0.9259 \\
$\mu$-Tools Reg LB & 300 points & 0.5051 & 25.00 \\
SS skew $\mu$ UB & 20 intervals & 1.1361 & {$[0.8,0.9]$} \\
SS skew $\mu$ LB & 20 intervals & 0.5182 & {$[10,100]$} \\
$\mu$-Tools UB & 100 points & 1.1322 & 0.8618 \\
\hline
\end{tabular}

search for the optimization-based lower bound algorithm is locating local optima. As in the case of the regularization-based lower bound search, the peak lower bound value obtained using this approach is within the interval $[10,100] \mathrm{rad} / \mathrm{s}$. Improved solutions may be obtained by relaxing the associated nonlinear constraint by reducing $\emptyset_{d}$. This is user-defined and must be chosen on a case-by-case basis. Regardless, a valid worst case problem perturbation is always obtained using the optimization-based solution, as shown in table 1 . The skew $\mu$ upper bound result is validated by performing a very dense frequency sweep of 100 grid points for the interval $[0.8,1] \mathrm{rad} / \mathrm{s}$. The peak value is indicated by an asterisk * in figure 3 and exceeds unity. It is important to emphasize that this result does not and will not exceed the skew $\mu$ upper bound result. The added benefit of this frequency interval or bounded approach is that it is far more computationally efficient than any frequency sweep. All key results obtained from each approach are shown in table 2 .

\section{CONCLUSIONS}

An overview of existing algorithms to determine lower bound solutions on real $\mu$ were outlined. The basic drawback with all of the approaches is that even if an improved lower bound is obtained, they are all based on a grid-based search and therefore are unreliable due to the discontinuous nature of real $\mu$. Instead transformation of the original $\mu$ problem into a skew $\mu$ where the peak value of $\mu$ is sought counteracts the discontinuity issue. It was shown for a MIMO mass-spring-damper example that both skew $\mu$ algorithms using a frequency bounded state-space formulation return more reliable and safe results than any conventional gridbased $\mu$ analysis approach.

\section{REFERENCES}

Balas, G., J. Doyle, K. Glover, A. Packard and R. Smith (1998). $\mu$-Analysis and Synthesis Toolbox User's Guide. The MathWorks Inc.

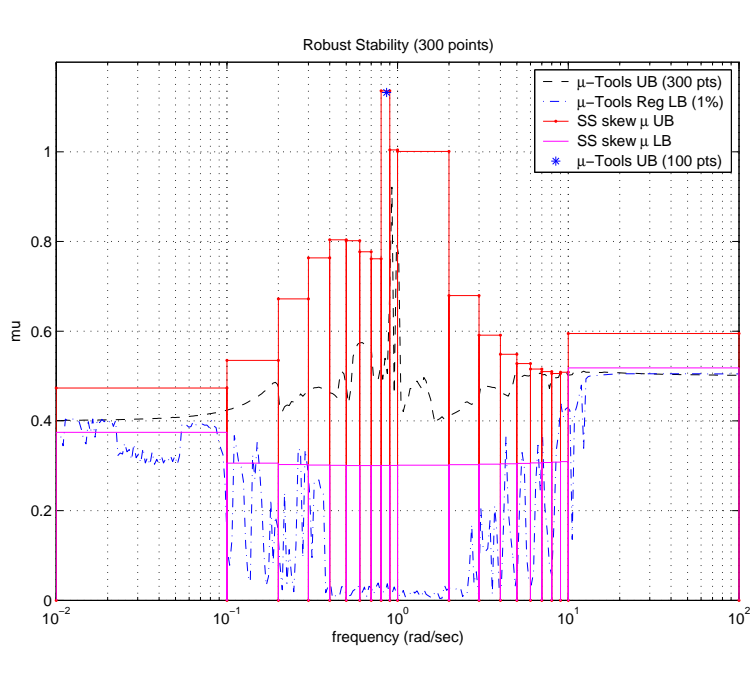

Fig. 3. Robust stability $\mu$-Tools bounds

Barmish, B.R., P.P. Khargonekar, Z.C. Shi and R. Tempo (1990). Robustness margin need not be a continuous function of the problem data. Systems and Control Letters 15, 91-98.

Branch, M.A. and A. Grace (1996). Optimization Toolbox. The MathWorks Inc.

Dailey, R. (1990). A new algorithm for the real structured singular value. In: Proc. of the ACC. pp. 3036-3040.

Doyle, J.C. (1982). Analysis of feedback systems with structured uncertainties. In: IEE Proceedings. Vol. 6. Chap. 129, pp. 242-250.

Doyle, J.C. and G. Stein (1981). Multivariable feedback design: concepts for a classical/modern synthesis. IEEE Transactions on Automatic Control 26, 4-16.

Halton, M.K. (2004). New structured singular value based robustness analysis tools with automotive applications. $\mathrm{PhD}$ Thesis. University of Limerick.

Holland, R., P. Young and C. Zhu (2003). Development of a skew $\mu$ upper bound. In: Proc. of ECC. pp. 349-354.

Holohan, A.M. and M.G. Safonov (1993). Some counterexamples in robust stability theory. Systems and Control Letters pp. 95-102.

Iordanov, P.I. (2003). Robust analysis and synthesis of systems subject to parameter uncertainty using the structured singular value. PhD Thesis. University of Limerick.

Packard, A. and P. Pandey (1993). Continuity properties of the real/complex structured singular value. IEEE Trans. Automatic Control 3, 415-428.

Sideris, A. (1992). Elimination of frequency search from robustness tests. IEEE Trans. Automatic Control 10, 1635-1640.

Young, P.M. and J.C. Doyle (1997). A lower bound for the mixed $\mu$ problem. IEEE Trans. Automatic Control pp. 123-128. 